\title{
The power of zero calcium score: Is there a need for improvement?
}

\author{
Marius Reto Bigler, $M D,^{a}$ and Christoph Gräni, $M D, P h D^{a}$ \\ a Department of Cardiology, Inselspital, Bern University Hospital, University of Bern, Bern, \\ Switzerland
}

Received Jul 30, 2020; accepted Jul 30, 2020

doi: $10.1007 / \mathrm{s} 12350-020-02326-7$

\section{See related article, pp. 323-333}

The early detection of coronary artery disease (CAD) is key for effective therapy decision-making to lower morbidity and mortality. Thus, the cardiovascular community made huge efforts to find optimal "gatekeeper" methods. A promising tool is the coronary artery calcium score (CACS), also called Agatston score, ${ }^{1}$ which allows visualization and quantitative assessment of calcified coronary atherosclerosis in native computed tomography, without the need of a needle or contrast agent. ${ }^{2,3}$ Thirty years after its introduction $^{1}$, CACS has emerged as the most predictive single cardiovascular risk marker in asymptomatic individuals, allowing preventive treatment modification in a selected population with borderline-to-intermediate atherosclerotic cardiovascular disease (ASCVD) risk (i.e., estimated risk of 5-20\%). ${ }^{4}$ Consequently, its application has been implemented by the guidelines ${ }^{5}$ in asymptomatic individuals. However, CACS is not (yet) recommended in symptomatic patients, although evidence exist of its excellent risk prediction, as recently shown by a meta-analysis of 34,000 patients with stable chest pain. ${ }^{6}$

Advantage of CACS is the following: It is a fast, low-cost, low-radiation test that allows in the absence of calcifications the exclusion of CAD with a high sensitivity (also known as "the power of zero"'). Therefore,

Funding None.

Reprint requests: Christoph Gräni, MD, PhD, Department of Cardiology, Inselspital, Bern University Hospital, University of Bern, Freiburgstrasse 18, 3010 Bern, Switzerland; christoph.graeni@ insel.ch

J Nucl Cardiol 2022;29:334-6.

$1071-3581 / \$ 34.00$

Copyright (C) 2020 American Society of Nuclear Cardiology.
CACS represents an ideal CAD—rule out test with a high negative predictive value, and can reclassify individuals in a high number. However, there are several shortcomings of the CACS. As stated by David E. Newby, "coronary artery calcification is a proxy of disease because it is induced in response to atherosclerosis" and represent an adaptive healing response of the body to limit the atherosclerotic disease. ${ }^{7}$ Are we too late in the process of imaging CAD by using CACS? We might indeed miss the early phase of CAD, and the most vulnerable plaques, the formation of non-calcified plaques (also known as "low-attenuation plaques" or "soft plaques") in a native computed tomography. In addition, stenosis grading is not possible, no information on myocardial perfusion is available, and therefore, CACS does not allow the assessment of hemodynamic relevance of the calcified plaques or a possible microvascular disease. Presence and extent of CACS, therefore, rather represent a surrogate for the atherosclerotic plaque burden and does not give any information on the ischemic impact on the myocardium level. Thus, several studies tried to further improve the diagnostic and prognostic value of CACS by adding clinical, anatomical (coronary computed tomography angiography (CCTA)), or functional testing.

In this issue of the Journal of Nuclear Cardiology, Megna et al., ${ }^{8}$ analyzed a large cohort of 1422 consecutive patients with zero calcium score (ZCS), who underwent ${ }^{82}$ Rubidium Positron emission tomography (PET)/CT for the evaluation of suspected CAD. The aim was to assess if commonly used risk scores for the prediction of obstructive CAD (i.e., Genders ${ }^{9}$ and Alshahrani ${ }^{10}$ ) are also useful to determine the probability of an abnormal stress myocardial perfusion imaging (MPI) study (i.e., relative and absolute myocardial blood flow) in this specific cohort. However, in the current study, the available clinical models failed to accurately predict an abnormal MPI among patients with a ZCS. 
In general, the prognosis of patients with ZCS is excellent. Considering the study of Mittal et al., none of the patients with ZCS $(n=1928)$ died of a coronary event over a 13-year follow-up period (mean $=5.2 \pm$ 2.8 years), resulting in a negative predictive value of 99.5\%. ${ }^{11}$ Similar results were found in three other clinical trials (CONFIRM, ${ }^{12}$ PROMISE, ${ }^{13}$ CRES$\mathrm{CENT}^{14}$ ), demonstrating the robustness of the power of zero. However, in reverse, another study showed that the annualized adjusted major adverse cardiovascular events were significantly different in the presence of impaired absolute myocardial perfusion reserve (MPR) among patients with ZCS $(1.4 \%$ vs $5.2 \%, P=0.03) .{ }^{15}$ Therefore, from a clinical point of view, the prediction of an abnormal MPR would provide valuable information about not only the correct underlying pathology but also the prognosis. It has been shown, that patients without CAD but with reduced MPR have similar adverse outcome rates as patients with $\mathrm{CAD}^{16}$ and absolute myocardial perfusion reserve is seen as an important independent outcome predictor. ${ }^{17}$ The study by Megna et al. could demonstrate that despite a CACS of zero, $17 \%$ had reduced MPR and 9\% showing ischemia (relative myocardial perfusion) according to the summed difference score. Since relative and absolute myocardial perfusion is usually multifaceted and can be provoked by $\mathrm{CAD}$ and/or microvascular disease, the etiology of the abnormal results remains unknown in this cohort. In a recent published article by Bittner et al., ${ }^{18}$ they demonstrated that frequency of obstructive CAD ( $\geq 70 \%$ stenosis in one major vessel/branch or $\geq 50 \%$ stenosis of left main) assessed by CCTA in patient cohort of ZCS is rare $(0.7 \%)$. Given a similar population in both studies, we can assume a similar frequency of obstructive CAD. Pursuing these considerations, most of the documented altered MPI studies in the current study are mostly due to microvascular disease, and in less frequent cases due to an obstructive CAD. Thus, this represents most probably a population where the management strategy remains unclear and evidence-based guidelines are widely missing. ${ }^{17}$

In conclusion, the present study by Megna et al. showed that the prevalence of impaired relative and absolute myocardial perfusion in a population with ZCS is not negligible and that clinical risk scores failed to improve prediction of reduced MPR or ischemia. This is an important finding with possible clinical consequences; however, the underlying cause of impaired myocardial perfusion in this cohort is unknown. First, better clinical tools are needed to narrow down the population for the selection of additional costly downstream testing and second, discrimination of a possible underlying obstructive or non-obstructive CAD and/or coronary microvascular dysfunction is needed to provide optimal diagnostics for management decisions. Beside the aim to establish the diagnosis, it is important to note that based on the comprehensive available prognostic data of ZCS, the value of additional testing for improving outcome prediction in such a population is unclear.

\section{Disclosures}

The authors have indicated that they have no financial conflict of interest.

\section{References}

1. Agatston AS et al (1990) Quantification of coronary artery calcium using ultrafast computed tomography. J Am Coll Cardiol 15:827832

2. Yakupoglu HY, Gräni C (2019) Role of imaging in primary prevention: Calcium score is a robust and cost-efficient risk modifier. Swiss Med Wkly 149:w20183

3. Gräni $\mathrm{C}$ et al (2018) Ultra-low-dose coronary artery calcium scoring using novel scoring thresholds for low tube voltage protocols: A pilot study. Eur Heart J 19:1362-1371

4. Greenland P et al (2018) Coronary calcium score and cardiovascular risk. J Am Coll Cardiol 72:434-447

5. Hecht $\mathrm{H}$ et al (2017) Clinical indications for coronary artery calcium scoring in asymptomatic patients: Expert consensus statement from the Society of Cardiovascular Computed Tomography. J Cardiovasc Comput Tomogr 11:157-168

6. Lo-Kioeng-Shioe MS et al (2020) Prognostic value of coronary artery calcium score in symptomatic individuals: A meta-analysis of 34,000 subjects. Int J Cardiol 299:56-62

7. Newby DE (2017) Computed tomography or functional stress testing for the prediction of risk: Can i have my cake and eat it? Circulation 136:2006-2008

8. Megna $\mathrm{R}$ et al (2020) Diagnostic value of clinical risk scores for predicting normal stress myocardial perfusion imaging in subjects without coronary artery calcium. J Nucl Cardiol. https://doi.org/ 10.1007/s12350-020-02247-5

9. Genders TS et al (2012) Prediction model to estimate presence of coronary artery disease: Retrospective pooled analysis of existing cohorts. BMJ 344:e3485

10. Alshahrani AM et al (2019) Point of care clinical risk score to improve the negative diagnostic utility of an Agatston score of zero: Averting the need for coronary computed tomography angiography. Circ Cardiovasc Imaging 12:e008737

11. Mittal TK et al (2017) Prevalence of obstructive coronary artery disease and prognosis in patients with stable symptoms and a zerocoronary calcium score. Eur Heart J Cardiovasc Imaging 18:922929

12. Cho I et al (2012) Coronary computed tomographic angiography and risk of all-cause mortality and nonfatal myocardial infarction in subjects without chest pain syndrome from the CONFIRM Registry (coronary CT angiography evaluation for clinical outcomes: An international multicenter registry). Circulation 126:304-313

13. Budoff MJ et al (2017) Prognostic value of coronary artery calcium in the PROMISE study (Prospective Multicenter Imaging Study for Evaluation of Chest Pain). Circulation 136:1993-2005 
14. Lubbers $\mathrm{M}$ et al (2016) Calcium imaging and selective computed tomography angiography in comparison to functional testing for suspected coronary artery disease: The multicentre, randomized CRESCENT trial. Eur Heart J 37:1232-1243

15. Naya M et al (2013) Prognostic interplay of coronary artery calcification and underlying vascular dysfunction in patients with suspected coronary artery disease. J Am Coll Cardiol 61:20982106

16. Murthy VL et al (2012) Association between coronary vascular dysfunction and cardiac mortality in patients with and without diabetes mellitus. Circulation 126:1858-1868
17. Bairey Merz CN et al (2017) Ischemia and no obstructive coronary artery disease (INOCA): Developing evidence-based therapies and research agenda for the next decade. Circulation 135:1075-1092

18. Bittner DO et al (2020) Prognostic value of coronary CTA in stable chest pain: CAD-RADS, CAC, and cardiovascular events in PROMISE. JACC: Cardiovasc Imaging 13:1534-1545

Publisher's Note Springer Nature remains neutral with regard to jurisdictional claims in published maps and institutional affiliations. 